\title{
Coulisses
}

Revue de théâtre

\section{L'opéra chinois et Le Roi Lear}

Une production en $2001 \mathrm{du}$ CLT, « Contemporary Legend Theatre » de Taïwan

\section{Françoise Quillet}

\section{(2) OpenEdition}

12 Journals

Édition électronique

URL : https://journals.openedition.org/coulisses/791

DOI : $10.4000 /$ coulisses. 791

ISSN : 2546-9460

Éditeur

Presses universitaires de Franche-Comté

\section{Édition imprimée}

Date de publication : 31 décembre 2010

Pagination : 53-64

ISBN : 978-2-84867-302-8

ISSN : 1150-594X

\section{Référence électronique}

Françoise Quillet, "L'opéra chinois et Le Roi Lear », Coulisses [En ligne], 41 | Automne 2010, mis en ligne le 30 novembre 2016, consulté le 29 décembre 2022. URL : http://journals.openedition.org/ coulisses/791; DOI : https://doi.org/10.4000/coulisses.791 


\title{
L'opéra chinois et Le Roi Lear
}

\author{
Une production en $2001 \mathrm{du}$ CLT, «Contemporary Legend Theatre » de \\ Taïwan
}

\section{Françoise Quillet}

1 Si « le monde est un théâtre », quel théâtre pour ce monde qui apparaît en ce début de $\mathrm{xxI}^{\mathrm{e}}$ siècle comme celui de la fracture, de la brisure, du fragment? Ce qui définit la scène moderne n'est plus l'autorité d'une forme qui, jadis, cristallisait la pratique théâtrale de toute une société, mais la mobilité des formes. L'artiste crée souvent audelà des bornes qui limitent le système bien défini de sa culture d'origine. Cette ouverture apparaît comme indissociablement liée à la régénération, au renouvellement de la création. Cette recherche n'est pas un phénomène nouveau, les créateurs ayant depuis longtemps pratiqué la «contamination» avec des œuvres venues de «l'ailleurs» mais elle s'intensifie depuis une cinquantaine d'années. Les avant-gardes européennes, à la recherche de formes nouvelles, s'intéressent aux traditions théâtrales de l'Extrême-Orient, détentrices, pour elles, d'un langage formulé avec rigueur et transmis avec fidélité. Les avant-gardes asiatiques s'emparent, de leur côté, des possibilités qu'offrent le drame réaliste et le théâtre d'origine occidental. Le grand maitre indien de Kathakali, Sadanam P.V Balakrishnan, adapte Le Cid, Yukio Ninagaura et Tadashi Suzuki mettent en scène des tragédies grecques, des pièces de Shakespeare et de Tchékhov.

2 La question qui se pose alors est de savoir si le rapport, de toute évidence, productif, du théâtre d'une culture avec des éléments de cultures théâtrales étrangères remplit dans chaque cas des fonctions très spécifiques qui ne peuvent pas être comparées les unes aux autres ou bien si la similitude des procédés renvoie à des principes communs qui laissent augurer une comparaison significative et féconde de tous ces phénomènes. Pour répondre à cette question, nous prendrons un exemple, celui de l'adaptation en 2001 du Roi Lear de Shakespeare, par l'acteur, dramaturge, Taïwanais, Wu Hsing-Kuo. Son entreprise fait partie d'un projet global de transformation de l'opéra chinois, au contact des techniques de jeu et de textes occidentaux. 


\section{Le contexte historique} en Chine. Shakespeare a été le dramaturge occidental le plus précocement introduit. La première apparition de son nom en chinois date de 1856 dans la traduction des Annales de Grande Bretagne publiée par le Collège de Mohai à Shanghai ${ }^{1}$. Objet d'une admiration profonde dans l'élite chinoise pendant plusieurs générations, il constitue le cas d'acculturation le plus ancien sans doute et le plus riche de l'histoire pré-communiste de la Chine. La réception de Shakespeare en Chine a cependant été complexe.

Dans les années 1910, premier âge d'or de la réception de Shakespeare en Chine, on trouve, à travers de nombreuses mises en scène, une volonté de sinisation de Shakespeare. Le nom des pièces est adapté en chinois, le thème central est déplacé, la structure de la représentation, transformée. Ce ne sont pas les différents aspects de l'âme humaine qui sont analysés à travers le destin d'individus mais les rapports sociaux. Ces perspectives changent de 1937 à 1945, lors de la guerre contre le Japon. Shakespeare est alors adapté dans une perspective plus littéraire et théâtrale que sociale. Mais les valeurs confucéennes restent les sources d'interprétation dominantes, métamorphosant l'esprit du dramaturge. Par la suite, de 1949 à 1966, les mises en scène de Shakespeare se font sous la direction de spécialistes soviétiques en fonction des théories stanislavskiennes de la représentation. Il faut attendre les années 1980, lorsque la Chine s'ouvre à l'Occident, pour voir une publication des Euvres complètes de Shakespeare. En 1984 seront créées conjointement une Association Shakespeare et le premier festival national sur Shakespeare. On assiste alors à la volonté de revaloriser la tradition théâtrale chinoise à travers un Shakespeare sinisé.

Lorsqu'en 2002 l'acteur Taïwanais, Wu Hsing-Kuo, adapte Le Roi Lear, il s'inscrit donc dans ce qui est devenu depuis un peu moins de deux siècles une "tradition». Son adaptation n'obéit pas aux critères habituels, ce qui en fait sa force et sa modernité. Nous allons donc tenter de rendre compte de la complexité et de la nature provocante de son travail de transposition culturelle. Afin d'éclairer le projet esthétique de $\mathrm{Wu}$ Hsing-Kuo, il est nécessaire de dire quelques mots sur la démarche qui a été la sienne.

\section{Le Contemporary Legend Theater ou comment l'opéra classique chinois peut devenir un théâtre moderne}

Dés l'âge de onze ans, il entre à l'école de l'opéra chinois, Fu-Hsing. Il y restera huit années, et se spécialisera dans les rôles masculins d'arts martiaux, Wu sheng. Après ces années d'apprentissage de l'opéra chinois, il intègre l'université, plus précisément le département de Théâtre de l'Université de la Culture Chinoise. À sa sortie, il devient le danseur principal d'une grande Compagnie de danse à Taïwan, le Cloud Gate Dance Theater. En 1977, il est membre de la Compagnie d'Opéra Chinois, Lu-Kuang. Il élargit alors son répertoire et se met à interpréter une grande variété de rôles pour lesquels il reçoit plusieurs récompenses dont le prix du meilleur acteur, Military Golden. Grâce à une bourse du Conseil de la Culture asiatique, il part étudier à New York.

7 Homme de théâtre talentueux, formé à l'école de l'opéra chinois, plus précisément de l'opéra de Pékin, danseur reconnu, Wu Hsing-Kuo est aussi un célèbre acteur de cinéma. Il a joué aux côtés de Jacky Chan et de Gong Li, et a été l'acteur principal de

Coulisses, 41 | Automne 2010 
Eighteen de Ho Ping, et de Green Snake de Tsui Hark. Il a reçu de nombreuses distinctions dont le prix du meilleur acteur, le Hong Kong Film Award, pour son rôle dans The Temptation of a Monk réalisé par Clara Law, en 1994.

8 Mais la plus grande aventure est pour Wu Hsing-Kuo la création en 1986 de la Compagnie, «Le Contemporary Legend Theatre » (CLT), dont il est à la fois le directeur artistique, le metteur en scène, le chorégraphe et l'acteur principal. Cette Compagnie réunit des acteurs de l'opéra de Pékin, conscients, comme lui, du déclin de l'opéra chinois classique. Son objectif est d'intégrer l'opéra chinois au théâtre moderne en puisant dans toutes les formes du répertoire, dans les pièces classiques et contemporaines chinoises et occidentales. C'est ainsi que depuis 1986 quatre pièces ont été adaptées de Shakespeare. Désir de royaume, une adaptation de Macbeth, Hamlet sous le nom Guerre et Éternité, Le Roi Lear en 2001 et La Tempête en 2004. Quatre autres ont été inspirées par des tragédies grecques, Médée en 1993 et L'Orestie en 1995. En 2005, année de la célébration du centenaire de Samuel Beckett, Wu Hsing-Kuo présente En Attendant Godot. Dans ses mises en scène il fait fusionner les techniques de l'acteur d'opéra chinois, c'est-à-dire le chant, la récitation, la danse, les combats acrobatiques et celles de l'acteur occidental, n'hésitant pas à insérer effets spéciaux et références au cinéma contemporain.

9 «L'héritage n'est plus suffisant, nous devons créer de nouveaux genres qui deviendront un nouveau patrimoine. Il faut changer la tradition, la moderniser », ces propos de Wu Hsing-Kuo le placent d'emblée comme un des grands réformateurs non seulement de la scène chinoise mais de la scène mondiale. Comment être à la fois fidèle à la forme artistique de l'opéra de Pékin et vouloir être le moteur du renouvellement de ses formes? Pour répondre à cette question, nous allons voir comment Wu Hsing-Kuo procède dans Le Roi Lear.

\section{La création du Roi Lear : un enfantement difficile}

Lorsque M. Ninagawa voit l'interprétation de Wu Hsing-Kuo dans Le Royaume du désir au Japon, en 1993, il lui déclare «Wu Hsing-Kuo, vous êtes le roi Lear ! » et lui suggère de jouer le rôle du roi Lear. Quelques années plus tard, en 1998, Ariane Mnouchkine qui assiste à la représentation du Royaume du désir au Festival d'Avignon l'invite en France pour diriger un atelier. C'est une époque difficile. Wu Hsing-Kuo n'a plus de budget pour entreprendre de nouveaux projets, de nombreux artistes ont quitté sa Compagnie, l'opéra de Pékin, même au travers les nouvelles pièces représentées, n'est plus soutenu par le gouvernement. La situation politique difficile entre Taïwan et la République populaire de Chine fait que tout élément « chinois » dans les arts est marginalisé voire rejeté. «Malgré nos efforts pour créer un opéra de Pékin nouveau, l'art que je professe était considéré comme de Pékin, à cause du nom².» Abandonné de tous, voire trahi par beaucoup, Wu Hsing-Kuo se sent alors comme le roi Lear. Il présente à Ariane Mnouchkine son adaptation du Roi Lear. Et elle insiste qu'il la mette en scène chez lui. De retour à Taïwan, estimant que Le Contemporary Legend Theater qu'il avait monté puis dissout devait exister, même s'il en était l'unique membre, Wu Hsing-Kuo fait revivre sa Compagnie. Grâce au directeur du Novel Hall de Taipei, Ku Huai-Chun, la première représentation du Roi Lear a lieu en 2001. Cette création ouvre une nouvelle voie expérimentale et contribue à la naissance en 2005 de l'adaptation de En Attendant Godot. 
11 La pièce de Shakespeare est revue et transformée par Wu Hsing-Kuo. Tout comme Lear a été banni, chassé et même réduit au silence par ses deux filles, comme Gloucester est traité par son fils bâtard, Wu Hsing-Kuo et son art ont été mal compris. La pièce est construite autour du parallèle entre le roi Lear et l'acteur Wu Hsing-Kuo. Ce dernier décide de faire un spectacle solo. Il réduit le nombre des personnages et simplifie l'intrigue. Nous allons rappeler brièvement l'intrigue shakespearienne afin d'éclairer le travail de Wu Hsing-Kuo.

\section{L'intrigue shakespearienne}

12 Le roi Lear décide de partager son royaume entre ses trois filles, Régane (épouse de Cornouailles), Goneril (épouse d'Albany) et Cordélia (dont le roi de France et le duc de Bourgogne se disputent la main). L'action est censée se dérouler dans une Grande Bretagne obscure et mythique d'il y a quelques milliers d'années, mais Shakespeare n'est pas à un anachronisme près, ce qui de plus facilite l'adaptation de la pièce. Lear promet la part la plus belle de son royaume à la fille qui saura le mieux exprimer son amour pour lui. Cordélia refuse de s'abaisser à flatter son père, comme ses sœurs, bien qu'elle l'aime profondément. Celui-ci, furieux et dépité de cette attitude, la déshérite et la chasse. Elle, cependant, part pour la France, fiancée à son roi. Un proche du roi Lear est également banni, le comte de Kent, qui a pris sa défense. Déguisé, il reviendra veiller sur son ancien maître. Ensuite, le roi, rejeté par Régane et Goneril, qui refusent de l'héberger comme cela a été convenu, se rend compte de son erreur et, désespéré, va errer sur la lande avec son bouffon, où il est rejoint par Kent, qui le persuade de s'abriter.

Une seconde intrigue se déroule parallèlement, toujours sur le thème de l'amour filial. Le comte de Gloucester a deux fils, Edmond (illégitime) et Edgar (légitime). Edmond fait croire à Edgar que sa vie est en danger et à son père qu'Edgar complote contre lui. Edgar s'enfuit et se déguise en Pauvre Tom, mendiant fou. Sur la lande Lear rencontre Edgar, avec qui il converse en délirant, et aussi Gloucester, qui ne reconnaît pas son fils et qui les emmène se mettre à l'abri.

14 Sur dénonciation d'Edmond, Régane et Cornouailles accusent Gloucester de trahison et lui font crever les yeux. Tandis que Goneril, accompagnée d'Edmond, va dire à Albany de lever une armée pour combattre celle que Cordélia amène de France pour secourir son père, on apprend la mort de Cornouailles. Toujours accompagné d'un Edgar déguisé, Gloucester, aveugle, rencontre Lear, fou, et dans une scène extraordinaire (IV. $\mathrm{VI}$ ), ces deux grands blessés parlent de tout et de rien, de la justice, de l'amour, de la souffrance et de la mort. Ensuite, Lear, réconcilié avec Cordélia, lui demande pardon. Mais lors de la bataille, l'armée de Lear et de Cordélia est défaite, et ils sont fait prisonniers par les troupes d'Albany et d'Edmond. La rivalité entre les deux sœurs, qui se disputent les faveurs d'Edmond, éclate au grand jour. Edgar, masqué, provoque Edmond en duel et le tue. Régane meurt empoisonnée par Goneril, qui se suicide. Lear entre, portant dans ses bras Cordélia, morte, pendue sur ordre d'Edmond. Gloucester est déjà mort, le cœur brisé, et le roi Lear meurt à son tour, penché au-dessus du corps de sa fille.

15 Le Roi Lear du Contemporary Legend Theater est plus qu'une transformation du Roi Lear, nous avons affaire à une mutation complète. Wu Hsing-Kuo en est le dramaturge, le metteur en scène, le chorégraphe et le seul interprète. 


\section{Une intrigue transformée par Wu Hsing-Kuo : un drame en trois actes aux titres révélateurs, " The Play, Playing, a Player »}

$16 \mathrm{Wu}$ Hsing-Kuo restructure la pièce en trois actes. Chaque acte, comme dans l'opéra chinois porte un titre : la pièce (The Play), le jeu (Playing), un acteur (A Player). Dans cette recomposition de la pièce se rejoignent le drame du Roi Lear et l'interrogation d'un acteur taïwanais sur son art. L'écriture dramatique s'émancipe aussi bien de l'écriture shakespearienne que de celle d'un opéra chinois. Elle invente, comme l'écriture scénique, sa propre forme entre Asie et Occident.

\section{Acte 1 La pièce (The play) ou la folie de Lear}

Rôle : Roi Lear

Cet acte est consacré au roi Lear et à son interprète, Wu Hsing-Kuo. Au commencement le roi Lear erre, seul, sur la lande. Banni par ses deux cruelles filles, hanté par le remords, il court, crie et vocifère dans le désert, dans la tempête. Les éléments déchaînés lui donnent la réplique. Un rond de lumière, délimité par quatre statues aux membres mutilés - image du roi Lear qui a perdu sa raison? -, définit l'aire de jeu où évolue Lear, sorte de cage dont il n'arrive pas à s'extraire.

Dés ce début il y a détournement de la forme chinoise. Dans le théâtre classique chinois dès qu'un personnage entre en scène, il se présente lui-même avec son nom, son passé, ses ambitions. Ce procédé vient du passage de la troisième personne à la première personne quand on a repris des œuvres de la littérature orale-chantée-contée pour les adapter au théâtre. Ce n'est pas son identité ni ses ancêtres que le héros décline devant nous mais sa seule souffrance.

Il y a détournement également de la forme shakespearienne. La chronologie est bouleversée. La pièce ne commence pas comme celle de Shakespeare, par le partage du royaume et l'abdication du souverain mais par sa chute physique et spirituelle, corporelle et sociale. Lear perd l'esprit. Wu Hsing-Kuo nous dépeint sa folie, les multiples moments où l'identité de Lear se désagrège progressivement. Pour que l'homme soit entièrement nu, ou plutôt pour qu'il ne soit plus qu'un homme, il ne suffit pas de lui retirer son nom, sa situation sociale, de le dépouiller de son caractère, il faut le mutiler moralement et physiquement. Alors seulement il pourra demander qui il est. Un aveugle est un homme, un fou est un homme, un vieillard hébété est un homme, rien qu'un homme, qui souffre, essaie de donner sens et dignité à sa souffrance. Le son plaintif du dong xiao, sorte de flûte, annonce la fin tragique de la pièce. Les projecteurs suivent Wu Hsing-Kuo qui tantôt virevoltant, tantôt vacillant, habite le personnage du roi Lear.

Du drame de la figure mythique et légendaire de Lear, nous passons à l'évocation du drame d'un acteur taïwanais d'aujourd'hui. Alors que Lear, blessé par la flèche de l'ingratitude filiale - flèche dont il mime le lancer, dont il suit du regard la trajectoire et qui revient se planter en son cœur - s'écroule à terre, Wu Hsing-Kuo se relève. Il arrache les cheveux et la longue barbe de Lear, cette longue barbe blanche que l'acteur 
chinois porte pour symboliser un personnage âgé. Il dévoile sa propre tête, son propre visage. Puis il enlève le costume de Lear, le somptueux costume de l'opéra chinois, le lance pesamment sur scène tandis que les questions se bousculent : "Qui suis-je? Qui est Lear? Est-ce que Lear marche ainsi, parle ainsi?». Il replie le costume de Lear. L'interrogation sur son identité se poursuit : «Je désire savoir qui je suis. » Il redevient lui-même mais il continue à proférer la réplique de Lear : "Qui suis-je? Quelqu'un me reconnaît-il ?» Le spectateur se demande alors ce qu'il est vraiment: le fantôme de Lear, lui-même? Le spectateur hésite. L'identité devient "flottante». "Voyageur de l'espace et du temps, il relie le passé à l'avenir, l'Est à l'Ouest ${ }^{3}$ » disait Peter Brook de son acteur japonais Yoshi Oida. Ces mots conviennent parfaitement dans le cas présent. Wu Hsing-Kuo se joue de lui-même, de son rapport à cette intrigue, personnage parmi les personnages, commentant l'action puis disparaissant pour laisser place au roi tourmenté. Le voici, en effet, redevenu Lear : « Je suis Lear, j'étais destiné à être Lear ». Wu Hsing-Kuo redécouvre la solitude de Lear tandis que la tempête se déchaîne. La fumée envahit la scène, le tonnerre gronde, les statues s'effondrent. L'univers intérieur de Lear s'écroule. De ses décombres s'élèvent d'innombrables fantômes qui cernent de tumulte la tête du vieux roi.

\section{Acte II En jouant (Playing)}

Rôles : Le fou, le chien, les trois filles, le comte de Gloucester, Edmond, Edgar

21 En un retour en arrière, en une remontée chronologique qui n'est pas sans rappeler la remontée qu'opère le personnage du shite (spectre) dans le théâtre japonais, plus précisément dans le nô, lorsque le spectre revit devant le spectateur le moment qui a provoqué sa mort, le roi Lear va revivre les moments qui ont provoqué sa folie. La flûte, comme dans le nô, ouvre ce début d'acte.

Il ne s'agit alors ni de forme chinoise ni de forme shakespearienne mais d'un rapport lointain à la forme d'une autre culture, japonaise, cette fois. Cette forme est elle-même transformée puisque Lear n'est pas ici un fantôme. Mais, comme dans le nô, nous remontons à la source du "mal», de ce qui a provoqué sinon la mort physique du moins la mort spirituelle.

Wu Hsing-Kuo devient alors non plus une identité «flottante» mais «une mer d'identités » tour à tour, le fou, le chien, Goneril, Régane, Cordélia, Gloucester, Edmond et Edgar. Il revit le moment où il a décidé de partager son royaume entre ses trois filles, celui où il a renié Cordélia, ceux où il a été renié par ses deux filles.

24 À l'intrigue de Lear et de ses trois filles succède celle de Gloucester et de ses deux fils. L'acte se termine sur la mort de ce dernier. Gloucester, après qu'on lui a arraché les yeux, veut se jeter dans la mer du haut des falaises de Douvres. Son propre fils, qui fait semblant d'être fou, le guide. Tous deux ont atteint le fonds de la misère humaine, ou, si l'on préfère, le sommet de cette "pyramide du malheur", ainsi que Slowacki qualifiait Le Roi Lear. Edgar soutient Gloucester. Il lève haut le pied, fait semblant de grimper, Gloucester lui aussi soulève la jambe. Cette scène de pantomime, est transformée. Alors que la tempête gronde, qu'on entend le bruit de la mer déchaînée Gloucester monte, seul, sur le rocher qui n'est autre qu'une des statues de l'acte précédent renversées par la tempête, il s'exclame : 
Edgar? mon fils

J'étais mauvais, je suis damné

Mon fils, ce père part maintenant.

Gloucester se jette dans l'océan, du haut d'une falaise, sans que son fils n'ait fait un geste pour l'en empêcher. On n'entend plus alors que la fureur des vagues. Va-t-il, comme dans le nô, retrouver le repos tant recherché auprès d'Amida (nom japonais de Bouddha Amithabha, suprême Bouddha du " paradis de l'ouest ») ? Rien n'est moins sûr car rien de tel n'est dit ici.

Représenter cette mort c'est, pour Wu Hsing-Kuo, représenter indirectement celle de son Maître. Jouer Edgar c'est se représenter lui-même :

Ma situation n'est pas différente de celle d'Edgar. Edgar est honnête, il aime son père. Toutefois il est mal compris et doit fuir. Quand je chante les airs d'Edgar, je me sens très proche du personnage. [...] Je me souviens du jour où mon Maître m'apprenait des techniques de jeu et me battait avec un bâton. J'étais déjà enseignant. J'ai saisi son bâton et lui ai dit : « Maître, pourquoi me battez-vous ? Il existe d'autres moyens d'enseigner. Bien que j'aie prononcé ces mots de façon très respectueuse, mon Maître ne m'a plus jamais parlé ${ }^{4} »$.

La mort du père d'Edgar fait écho ainsi à celle du Maître et au rêve qu'eut Wu HsingKuo :

Dans ce dernier mon maître voulait me tuer avec une épée, j'ai été contraint de la lui prendre et de le tuer. Ce rêve m'a vraiment choqué. Deux mois après mon retour à Taïwan, mon maître est mort subitement.

Au milieu de sa pièce, Wu Hsing-Kuo a placé l'histoire de Gloucester, résonance de son drame d'artiste qui remet en question la tradition, pour qui le théâtre est un lieu vivant dont le but est de concerner des gens d'aujourd'hui.

" Tuer le Maître, c'est tuer la tradition » proclame souvent Wu Hsing-Kuo. Il s'agit non de nier mais de transformer. Entre les deux actes, d'ailleurs, s'élève, une voix off, celle du conteur Wu Hsing-Kuo :

Mon père m'a donné une chance de le tuer mais je ne l'ai pas tué. Après cette rencontre père et fils vont renaître. C'est le moment le plus tendre du Roi Lear mais Lear est fou, arpentant la lande.

\section{Acte III Un acteur (A player)}

Rôle : Wu Hsing-Kuo

L'acte suivant commence sur une longue incantation. Deux musiciens parcourent la scène en une longue déambulation et psalmodient des chants bouddhiques. La référence à Amida et, pourrait-on dire, au nô se retrouve une fois encore. Ce cérémonial est-il destiné à Gloucester qui vient de perdre la vie, au roi Lear qui ne réapparaitra plus, à Wu Hsing-Kuo qui, en habit de moine bouddhiste, arrive sur scène en portant solennellement le costume de Lear, comme s'il portrait le corps défunt de Cordélia?

Il va psalmodier, à son tour, un regret sans fin, intégrant la vie de Lear au plus proche de sa mémoire personnelle, mêlant cette grande tragédie à l'histoire de la vie d'un acteur de l'opéra de Pékin, né et élevé à Taïwan.

Qui suis-je ? Je suis moi

Me cherchant, je pense 


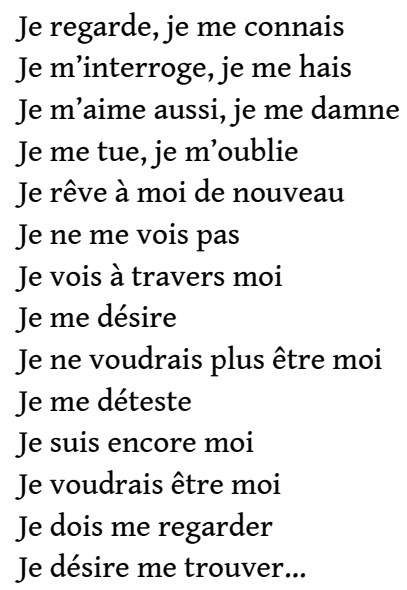

Portant le costume de Lear, il passe devant le rocher d'où s'est jeté Gloucester et s'exclame : «Ciel, pourquoi m'avez-vous envoyé cette cage? »

Toute ma vie pour garder la Beauté [...]

Quels instruments de torture ont façonné ma nature

Quelle haine a chassé mon amour

Lear, Lear, qu'est-ce qui m'est arrivé d'être Lear?

Cette pièce n'est pas, comme la pièce de Shakespeare, une tragédie, elle n'est pas comme une pièce d'opéra chinois, une comédie, elle ne finit pas comme un nô, par l'effacement du personnage dans les premières lueurs de l'aube. La fin n'est ni tragique, ni comique, pas vraiment apaisée. Y a-t-il d'ailleurs une fin? Wu Hsing-Kuo déposant le costume de Lear sur le sol non plus brutalement comme dans l'acte I mais avec respect, nous parle :

Qu'est-ce qui m'est arrivé d'être Lear? [...]

Il me reste une prison avec quatre murs.

Seul et tranquille je regarde la lune qui va et vient.

34 Au-delà du discours se dresse une voûte céleste dans laquelle planent des figures lumineuses. Au milieu d'elles, la lune, chargée des désirs humains, les éclaire tous. Cette lune, un des symboles fondamentaux des poètes chinois classiques, révèle le secret d'une nuit de mythe et de communion.

Commencée avec le tumulte du vent, le grondement de la tempête, le feu des éclairs, la pièce se termine sur cette vision qui se voudrait apaisée. Loin de se fixer sur un seul point, la lune a permis, à travers les multiples variations des êtres, ce grand voyage dans le temps entre l'extrême de l'Orient et l'extrême de l'Occident.

\section{Un renouvellement des formes scéniques}

Passant d'un costume à l'autre, du masculin au féminin, de l'élégance la plus raffinée à la violence la plus désespérée d'un roi abandonné, Wu Hsing-Kuo a interprété les personnages shakespeariens selon les codes de l'opéra chinois. En un clin d'œil, il s'est transformé en chou (le bouffon), sheng (le jeune homme), dan (la jeune femme), jing (le gredin), mo (l'homme d'âge mur), Lear devenu fou, Gloucester aux yeux crevés. Il a déclamé et chanté un Shakespeare traduit en chinois. Entouré de ses neuf musiciens qui rythment les gestes et les déplacements des personnages qui se créent successivement sous les yeux des spectateurs, Wu Hsing-Kuo ne déroge pas à l'art de l'acteur chinois qui est d'être acrobate, danseur et chanteur. Il n'épargne ni sa personne ni le 
spectateur. Il possède toutes les ressources de la voix, la plénitude du geste, l'ampleur du mouvement, la flexibilité et l'assurance du corps, la force du regard. Il n'y a pas de « grand» Shakespeare sans grand acteur, Wu Hsing-Kuo en est un assurément.

Les codes de l'opéra de Pékin sont revus et corrigés par ceux du théâtre occidental. En interprétant les trois filles, il retrouve la tradition élisabéthaine qui, en interdisant aux femmes de paraître sur scène, obligeait les acteurs à jouer les rôles féminins. La musique, comme dans l'opéra chinois, occupe ici une place essentielle mais réunit tonalités classiques chinoises, musique contemporaine et électronique. Les moments chantés, fine fleur de l'opéra chinois, n'occupent plus la première place. Cet art qui éblouit l'œil du spectateur occidental, provient de l'opéra chinois mais il est épuré, simplifié, comme le sont les costumes et les maquillages. Le somptueux costume de Lear n'est visible que dans l'acte 1 . La scène, sans table ni chaise, n'est pas la scène de l'opéra chinois. Le Roi Lear est joué sur un plateau au décor d'une beauté minimaliste.

Si la mise en scène s'inspire de l'opéra de Pékin, on y trouve aussi, ce qui en explique en partie, la modernité, de nombreux clins d'œil à l'expérience cinématographique. Chaque acte est composé comme un cadrage de film et opère par gros plans; la lumière qui cerne les silhouettes, met en valeur ces plans. Les trois actes se succèdent par fondus-enchaînés, l'obscurcissement signifie un saut dans le temps. Cette adaptation repose sur un montage d'instants de tension, sans temps morts, les séquences sont autant de grands tableaux dramatiques.

Son solo, inspiré de l'opéra de Pékin, nous entraîne loin de ses conventions ou plutôt les libère, créant des formes nouvelles qui ne renient pas mais transforment les anciennes, retrouvant le caractère spectaculaire que Shakespeare conférait au théâtre.

Wu Hsing-Kuo a appréhendé Le Roi Lear comme un texte vivant, toujours en mouvement Il a «tué » la tradition aussi bien occidentale que chinoise en ce qu'elle a de fixité, d'immobilisme :

Se débarrasser de la mémoire du théâtre [...] c'est aussi explorer les conditions d'une communication qui ne tienne pas compte des références culturelles, délivrer l'acteur de son conditionnement psychologique et social, c'est faire du non-savoir et de l'ouverture la qualité fondamentale non seulement de l'acteur et du spectateur mais de la quête elle-même ${ }^{5}$.

41 Il a su donner au Roi Lear, un des grands textes du théâtre occidental, une place originale dans un univers différent. Ecouter le sens d'une œuvre dans sa culture d'origine et la faire résonner en l'ouvrant à un autre horizon culturel, c'est, sans conteste, la réussite de cette adaptation. Ce Roi Lear est la révélation d'un Shakespeare qu'on pressent, dont on rêve mais qu'on ne voit que rarement sur une scène. Il en montre l'esprit et la modernité.

\section{Conclusion}

Pour répondre à notre question initiale, on peut dire que l'adaptation du Roi Lear par Wu Hsing-Kuo remplit une fonction bien spécifique dans la mesure où l'auteur acteur établit un rapport entre un personnage shakespearien et un acteur contemporain. Le problème dépasse alors celui de la rencontre des cultures, ou plus exactement, la question posée à une époque dans une culture est reprise, intériorisée et élargie. N'estce pas la marque de toute forme artistique réussie, qu'elle soit interculturelle ou non? 
theâtre différent s'invente qui, à Taïwan, à New York, à Paris, s'adressant à tous, bouleverse au plus profond chaque spectateur. Questionnant sans cesse le public avec cette demande lancinante "Qui suis-je? Qui peut me dire qui je suis?", cette adaptation du Roi Lear questionne l'essence du théâtre et le sens de l'existence.

LEAR

Me traites-tu de fou, gamin?

LE FOU

Tous les autres titres, tu les as gaspillés ; celui-là, tu étais né avec.

(Le Roi Lear, I, 4)

Nous naissons tous fous. Quelques-uns le demeurent.

(En Attendant Godot, II)

Que Wu Hsing-Kuo représente en 2005 En Attendant Godot ne saurait nous étonner.

\section{NOTES}

1. Ces informations proviennent de l'ouvrage de ZHANG Ning, L'Appropriation par la Chine du théâtre occidental Un autre sens de l'Occident, L'Harmattan, 1998.

2. Alexandre $\mathrm{CY}$ Huang, "Extrait d'une entrevue avec Wu Hsing-Kuo », in Shakespeare sur la scène chinoise 1839-2004, thèse de doctorat, Stanford University, 2004

3. Yoshi Oida, L'Acteur flottant, Actes Sud, 1992, p. 8.

4. Alexandre CY Huang, "Extrait d'une entrevue avec Wu Hsing-Kuo ", in Shakespeare sur la scène chinoise 1839-2004, thèse de doctorat, Stanford University, 2004.

5. Monique Borie, op.cit., p121.

INDEX

Palabras claves : opera chino, adaptación, Contemporary Legend Theater oeuvrecitee Roi Lear (Le)

Mots-clés : opéra chinois, adaptation, Contemporary Legend Theater

Palavras-chave : opera chinês, adaptação, Contemporary Legend Theater

Keywords : chinese opera, adaptation, Contemporary Legend Theater

\section{AUTEURS}

\section{FRANÇOISE QUILLET}

Université de Franche-Comté. Maître de conférences, Département des Arts du Spectacles 